\title{
AVERAGE CASE COMPLEXITY OF MULTIVARIATE INTEGRATION
}

\author{
H. WOŹNIAKOWSKI
}

\begin{abstract}
We study the average case complexity of multivariate integration for the class of continuous functions of $d$ variables equipped with the classical Wiener sheet measure. To derive the average case complexity one needs to obtain optimal sample points. This optimal design problem has long been open. All known designs guaranteeing average case error $\varepsilon$ lead to an exponential number of sample points, roughly $\Theta\left(\varepsilon^{-d}\right)$. For $d$ large this makes the problem intractable for even the fastest computers.

Yet good designs have to exist since the average case complexity is bounded by $O\left(\varepsilon^{-2}\right)$ as can be proven by considering the Monte-Carlo algorithm. We just did not know how to construct them.

In this paper we prove that optimal design is closely related to discrepancy theory which has been extensively studied for many years. Of particular importance for our purpose are papers by Roth $[10,11]$. This relation enables us to show that optimal sample points can be derived from Hammersley points. Extending the result of Roth [10] and using the recent result of Wasilkowski [19], we conclude that the average case complexity is $\Theta\left(\varepsilon^{-1}\left(\ln \varepsilon^{-1}\right)^{(d-1) / 2}\right)$.
\end{abstract}

\section{INTRODUCTION}

The approximate computation of multivariate integrals has been extensively studied in many papers, see [5-7, 17] for hundreds of references. We assume that multivariate integrals are approximated by evaluating integrands at finitely many sample points and by performing arithmetic operations and comparisons on real numbers. Assume that the cost of one integrand evaluation is $\mathbf{c}$, and that the cost of one arithmetic operation or comparison is taken as 1 . Usually $\mathbf{c} \gg 1$.

Received by the editors September 28, 1989.

1980 Mathematics Subject Classification(1985 Revision). Primary 65D30,41A55.

This research was supported in part by the National Science Foundation under Contract IRI-89-07215. 
We seek the computational complexity of multivariate integration, which is defined as the minimal cost of approximating multivariate integrals with error at most $\varepsilon$ for a given class $F$ of integrands, see [17].

How we define cost and error depends on the setting. In the worst case setting, the cost of an approximation is defined as

$$
\sup _{f \in F}\left(\mathbf{c} n_{1}(f)+n_{2}(f)\right),
$$

where $n_{1}(f)$ is the number of integrand evaluations and $n_{2}(f)$ is the number of arithmetic operations and comparisons needed to compute the approximation for $f \in F$. The error of the approximation is defined as the maximal absolute difference between the exact and approximate values of multivariate integrals over the class $F$. The computational complexity in the worst case setting is denoted by $\operatorname{comp}^{\text {wor }}(\varepsilon, F)$ and is known for many classes $F$. For example, if $F=W_{p}^{r, d}$ is the Sobolev class of real functions defined on the $d$-dimensional cube $D=[0,1]^{d}$ whose $r$ th distributional derivatives exist and are bounded in the $L_{p}$ norm by one, then for $p r>d$ we have $\operatorname{comp}^{\text {wor }}\left(\varepsilon, W_{p}^{r, d}\right)=\Theta\left(\mathbf{c} \varepsilon^{-d / r}\right)$, see [7] for a recent survey. For $d$ large relative to $r$, the worst case complexity is huge even for moderate $\varepsilon$.

In the average case setting, which is the focus of this paper, the cost and error of approximations are defined as the expected cost and error with respect to some probability measure on the class $F$. The computational complexity in the average case setting is denoted by $\operatorname{comp}^{\text {avg }}(\varepsilon, F)$. In contrast to the enormous literature for the worst case setting, the average case setting for multivariate integration has been studied in relatively few papers, see [1-4, 7-9, 12-20].

We report briefly on what is known about $\operatorname{comp}^{\text {avg }}(\varepsilon, F)$. For the scalar case $d=1$, let $F=C^{r}$ be the class of $r$ times continuously differentiable functions equipped with $r$-fold Wiener measure. Then

$$
\operatorname{comp}^{\text {avg }}\left(\varepsilon, C^{r}\right) \simeq \mathbf{c}\left(\alpha \varepsilon^{-1}\right)^{1 /(r+1)},
$$

where $\alpha=\sqrt{\left|B_{2 r+2}\right| /(2 r+2) !}$ and $B_{2 r+2}$ is a Bernoulli number. Furthermore, optimal sample points and how to optimally combine the integrand evaluations at these points are known, see [3, 15, 17]. 
For the multivariate case, $d \geq 1$, assume that $F=C_{d}$ is the class of real continuous functions defined on $D=[0,1]^{d}$ and equipped with the classical Wiener sheet measure $w$. That is, $w$ is Gaussian with mean zero and covariance kernel

$$
R(s, t) \stackrel{\text { def }}{=} \int_{C_{d}} f(s) f(t) w(d f)=\min (s, t) \stackrel{\text { def }}{=} \prod_{j=1}^{d} \min \left(s_{j}, t_{j}\right)
$$

for any vectors $s=\left[s_{1}, \ldots, s_{d}\right]$ and $t=\left[t_{1}, \ldots, t_{d}\right]$ from $D$.

Only upper and lower bounds are known for $\operatorname{comp}^{\text {avg }}\left(\varepsilon, C_{d}\right)$. Papageorgiou [8] established

$$
\begin{array}{r}
\operatorname{comp}^{\text {avg }}\left(\varepsilon, C_{d}\right)=\Omega\left(\mathbf{c} \varepsilon^{-1}\right) \text { and } \operatorname{comp}^{\text {avg }}\left(\varepsilon, C_{d}\right)=O\left(\mathbf{c} \varepsilon^{-2}\right), \\
\forall d \geq 1 .
\end{array}
$$

The proof of the upper bound is based on the Monte-Carlo algorithm using randomized sample points. Therefore it does not provide a constructive way to find deterministic sample points that achieve the bound $O\left(\mathbf{c} \varepsilon^{-2}\right)$.

To obtain the average case complexity, one needs to find optimal sample points, i.e., sample points which lead to minimal average case error. It is known that optimal sample points do not form a grid. That is, if one assumes that sample points form a grid

$$
\left\{\left[i_{1} h_{1}, \ldots, i_{d} h_{d}\right]: i_{j}=1, \ldots, m_{j}, j=1,2 \ldots, d\right\}
$$

with $h_{j}=m_{j}^{-1}$ for some integers $m_{j}$, then Papageorgiou and Wasilkowski [8,9], see also [20], showed that $\Theta\left(\varepsilon^{-d}\right)$ grid sample points are needed to achieve the average case error $\varepsilon$. For $d>$ 2 , this cost is worse than the upper bound in (1). This proves that grid points are a poor choice of sample points. Papageorgiou [8] slightly improved this result by constructing $\Theta\left(\varepsilon^{-\left(d^{2}-d+1\right) / d}\right)$ sample points for which the average case error is $\varepsilon$. For $d=2$, these sample points reduce to those proposed by Ylvisaker [20]. Thus, for $d=2$ we have an improved upper bound

$$
\operatorname{comp}^{\text {avg }}\left(\varepsilon, C_{d}\right)=O\left(\mathbf{c} \varepsilon^{-1.5}\right) .
$$

The average case complexity $\operatorname{comp}^{\text {avg }}\left(\varepsilon, C_{d}\right)$ has been unknown due to the difficulty of finding optimal sample points. In the statistical literature this is called the optimal design problem for multivariate integration, see [20]. Micchelli and Wahba [4] conjectured that Hammersley points (defined below) should lead to an 
optimal convergence rate $n^{-1}(\log n)^{d-1}$ which would imply that $\operatorname{comp}^{\text {avg }}\left(\varepsilon, C_{d}\right)$ is $\Theta\left(c \varepsilon^{-1}\left(\log \varepsilon^{-1}\right)^{d-1}\right)$. This form of the average case complexity has been also conjectured by Papageorgiou and Wasilkowski [9], based on their work on the approximation problem.

In this paper we show that the optimal sample points problem is related to the $L_{2}$ discrepancy (defined below). The discrepancy in $L_{2}$ and other norms has been extensively studied in the literature and deep relations with number theory have been established. In particular, it is known that Hammersley points lead to small discrepancy. The reader is referred to excellent surveys by Niederreiter [5, 6], where the background, history and about 500 references concerning discrepancy may be found.

The $L_{2}$ discrepancy has been studied by Roth $[10,11]$ who proved in 1954 that the $L_{2}$ disrepancy of $n$ points has to be at least of order $n^{-1}(\log n)^{(d-1) / 2}$, and in 1980 that this bound is sharp. Since the $L_{2}$ discrepancy is minimized by shifted Hammersley points, we conclude that optimal sample points are related to Hammersley points and that the average case complexity is $\Theta\left(\mathbf{c} \varepsilon^{-1}\left(\log \varepsilon^{-1}\right)^{(d-1) / 2}\right)$.

\section{MAIN RESUlT}

For $t=\left[t_{1}, \ldots, t_{d}\right] \in D=[0,1]^{d}$, define $[0, t)=\left[0, t_{1}\right) \times$ $\cdots \times\left[0, t_{d}\right)$. Let $\chi_{[0, t)}$ be the characteristic (indicator) function of $[0, t)$. For $z_{1}, \ldots z_{n} \in D$ define

$$
R_{n}\left(t ; z_{1}, \ldots, z_{n}\right)=n^{-1} \sum_{k=1}^{n} \chi_{[0, t)}\left(z_{k}\right)-t_{1} t_{2} \cdots t_{d}
$$

as the difference between the fraction of the points $z_{i}$ in $[0, t)$ and the volume of $[0, t)$. The $L_{2}$ discrepancy of $z_{1}, \ldots, z_{n}$ is defined as the $L_{2}$ norm of the function $R\left(\cdot ; z_{1}, \ldots, z_{n}\right)$. Roth $[10,11]$ proved that

$$
\inf _{z_{1}, \ldots, z_{n}}\left(\int_{D} R_{n}^{2}\left(t ; z_{1}, \ldots, z_{n}\right) d t\right)^{1 / 2}=\Theta\left(n^{-1}(\log n)^{(d-1) / 2}\right) .
$$

The points $z_{1}^{*}, \ldots, z_{n}^{*}$ with $L_{2}$ discrepancy of order $n^{-1}(\log n)^{(d-1) / 2}$ are related, as indicated below, to Hammersley points. As in $[5,11]$, let $p_{1}, p_{2}, \ldots, p_{d-1}$ be the first $(d-1)$ prime numbers. Any integer $k \geq 0$ can be uniquely represented 
as $k=\sum_{i=0}^{\lceil\log k\rceil} a_{i} p_{j}^{i}$ with integers $a_{i} \in\left[0, p_{j}-1\right]$. The radical inverse function $\phi_{p_{j}}$ is given as

$$
\phi_{p_{j}}(k)=\sum_{i=0}^{\lceil\log k\rceil} a_{i} p_{j}^{-i-1}
$$

The sequence $\left\{u_{k}\right\}$ of $(d-1)$ dimensional points for $k=0, \pm 1$, $\pm 2, \ldots$ is defined by

$$
u_{k}=\left[\phi_{p_{1}}(k), \phi_{p_{2}}(k), \ldots, \phi_{p_{d-1}}(k)\right], \quad k=0,1, \ldots, M-1,
$$

with $M=\left(p_{1} p_{2} \cdots p_{d-1}\right)^{\lceil\log n\rceil}$, and by $u_{k+M}=u_{k}, \forall k$. Then there exists a real number $t^{*}$ such that the $d$ dimensional points $z_{1}^{*}, \ldots, z_{n}^{*}$ are obtained by adding one component to the $(d-1)$ dimensional points $u_{k}$,

$$
\left\{z_{1}^{*}, \ldots, z_{n}^{*}\right\}=\left\{\left[\left(k+t^{*}\right) n^{-1}, u_{k}\right]: 0 \leq k+t^{*}<n\right\} .
$$

For $t^{*}=0$, they are Hammersley points. Thus, the points $z_{i}^{*}$ are obtained by adding $t^{*} / n$ to the first component of Hammersley points.

We are ready to show how the results on $L_{2}$ discrepancy can be used to derive optimal sample points and average case complexity. In what follows, we use the word "optimal" modulo a constant which may depend on $d$ but is independent of $\varepsilon$.

Let $n=\Theta\left(\varepsilon^{-1}\left(\log \varepsilon^{-1}\right)^{(d-1) / 2}\right)$ be chosen such that the $L_{2}$ discrepancy of the function $R_{n}\left(\cdot, z_{1}^{*}, \ldots, z_{n}^{*}\right)$ is at most $\varepsilon$. Letting $\overrightarrow{1}=[1,1, \ldots, 1]$, we define

$$
x_{k}^{*}=\overrightarrow{1}-z_{k}^{*}, \quad k=1,2, \ldots, n .
$$

We approximate the integral of $f$ from $C_{d}$ by the arithmetic mean of its values at $x_{k}^{*}$,

$$
I(f) \stackrel{\text { def }}{=} \int_{D} f(t) d t \sim U(f) \stackrel{\text { def }}{=} n^{-1} \sum_{k=1}^{n} f\left(x_{k}^{*}\right), \quad \forall f \in C_{d} .
$$

Clearly $\operatorname{cost}(U)$, the cost of computing $U(f)$, is $(\mathbf{c}+1) n$. The average case error of $U$ is defined as $\left(\int_{C_{d}}(I(f)-U(f))^{2} w(d f)\right)^{1 / 2}$. We summarize the main results as a

Theorem. (i) $\operatorname{comp}^{\mathrm{avg}}\left(\varepsilon, C_{d}\right)=\Theta\left(\mathbf{c} \varepsilon^{-1}\left(\log \varepsilon^{-1}\right)^{(d-1) / 2}\right)$,

(ii) $x_{1}^{*}, x_{2}^{*}, \ldots, x_{n}^{*}$ given by (4) are optimal sample points, 
(iii) $U$ given by (5) is optimal, i.e., the average case error of $U$ is at most $\varepsilon$ and

$$
\operatorname{cost}(U)=\Theta\left(\operatorname{comp}^{\text {avg }}\left(\varepsilon, C_{d}\right)\right) .
$$

Sketch of the proof. An upper bound on $\operatorname{comp}^{\text {avg }}\left(\varepsilon, C_{d}\right)$ is $\operatorname{cost}(U)$ provided we prove that the average case error of $U$ is at most $\varepsilon$. This will be done by exhibiting the identity which relates the average case error of $U$ with the $L_{2}$ discrepancy.

\section{Lemma 1.}

$$
\int_{C_{d}}(I(f)-U(f))^{2} w(d f)=\int_{D} R_{n}^{2}\left(t ; z_{1}^{*}, \ldots, z_{n}^{*}\right) d t .
$$

To prove Lemma 1, we use

$$
\int_{C_{d}} I^{2}(f) w(d f)=3^{-d}
$$

and

$$
\int_{C_{d}} I(f) f(t) w(d f)=\prod_{i=1}^{d} t_{i}\left(1-t_{i} / 2\right), \quad \forall t=\left[t_{1}, \ldots, t_{n}\right] .
$$

From these formulas we compute the left-hand side (LHS) of Lemma 1,

$$
\text { LHS }=3^{-d}-\frac{2}{n} \sum_{k=1}^{n} \prod_{i=1}^{d} x_{k, i}^{*}\left(1-x_{k, i}^{*} / 2\right)+\frac{1}{n^{2}} \sum_{k, p=1}^{n} \min \left(x_{k}^{*}, x_{p}^{*}\right) \text {, }
$$

where $x_{k}^{*}=\left[x_{k, 1}^{*}, \ldots, x_{k, d}^{*}\right]$. The right-hand side (RHS) of Lemma 1 can be directly computed,

$$
\begin{gathered}
\text { RHS }=\int_{D} t_{1}^{2} \cdots t_{d}^{2} d t-\frac{2}{n} \sum_{k=1}^{n} \int_{D} t_{1} \cdots t_{d} \chi_{[0, t)}\left(z_{k}^{*}\right) d t \\
+\frac{1}{n^{2}} \sum_{k, p=1}^{n} \int_{D} \chi_{[0, t)}\left(z_{k}^{*}\right) \chi_{[0, t)}\left(z_{p}^{*}\right) d t \\
=3^{-d}-\frac{2}{n} \sum_{k=1}^{n} \prod_{i=1}^{d} \frac{1-z_{k, i}^{*} 2}{2}+\frac{1}{n^{2}} \sum_{k, p=1}^{n} \prod_{i=1}^{d}\left(1-\max \left(z_{k, i}^{*}, z_{p, i}^{*}\right)\right) .
\end{gathered}
$$

We complete the proof of Lemma 1 by substituting $z_{k, i}^{*}=1-x_{k, i}^{*}$ and noting that $1-\max (1-a, 1-b)=\min (a, b)$.

Due to the choice of $n$ and the construction of $z_{i}^{*}$, the average case error of $U$ is at most $\varepsilon$. This completes the proof of the upper bound on $\operatorname{comp}^{\text {avg }}\left(\varepsilon, C_{d}\right)$. 
To derive a lower bound on $\operatorname{comp}^{\text {avg }}\left(\varepsilon, C_{d}\right)$, we use a proof technique from [17]. First take any nonadaptive sample points $x_{1}, \ldots, x_{n}$ from $D$. Since $w$ is Gaussian and the average case error is defined in the $L_{2}$ sense, it is known that the approximation $U^{*}$ with minimal average case error is the mean of the conditional probability, given $f\left(x_{1}\right), \ldots, f\left(x_{n}\right)$. Since the mean depends linearly on $f\left(x_{i}\right), U^{*}$ takes the form $U^{*}(f)=\sum_{k=1}^{n} c_{k} f\left(x_{k}\right), \forall f \in$ $C_{d}$, for some numbers $c_{k}$. Using similar calculations as in the proof of Lemma 1 we get

\section{Lemma 2.}

$$
\begin{aligned}
\int_{C_{d}} & \left(I(f)-U^{*}(f)\right)^{2} w(d f) \\
& =\int_{D}\left(\sum_{k=1}^{n} c_{k} \chi_{[0, t)}\left(\overrightarrow{1}-x_{k}\right)-t_{1} \cdots t_{d}\right)^{2} d t .
\end{aligned}
$$

It is possible to extend the proof of Roth [10] to show that the lower bound of (2) holds for all $c_{k}$. More precisely we have

Lemma 3. There exists a positive number $\gamma_{d}$ such that

$$
\int_{D}\left(\sum_{k=1}^{n} c_{k} \chi_{[0, t)}\left(\overrightarrow{1}-x_{k}\right)-t_{1} \cdots t_{d}\right)^{2} d t \geq \gamma_{d} n^{-2}(\log n)^{d-1}
$$

for all $n, c_{k}$ and $x_{k}$.

From Lemmas 2 and 3 we conclude that the average case error of $U^{*}$ is bounded by $\varepsilon$ only if $n=\Omega\left(\varepsilon^{-1}\left(\log \varepsilon^{-1}\right)^{(d-1) / 2}\right)$. Therefore the cost of approximating $I(f)$ with average case error $\varepsilon$ must be at least $\mathbf{c} n=\Omega\left(\mathbf{c} \varepsilon^{-1}\left(\log \varepsilon^{-1}\right)^{(d-1) / 2}\right)$.

Consider now adaptive sample points $x_{1}, x_{2}, \ldots, x_{n(f)}$. That is, the choice of $x_{i}$ may depend on the already computed $f\left(x_{1}\right)$, $\ldots, f\left(x_{i-1}\right)$, and the number $n(f)$ of sample points may also be adaptively chosen. For Gaussian measures adaption may help only by varying $n(f)$. We now draw on Wasilkowski's theorem, see [19], which states that adaption can help only by a multiplicative constant if the squares of the minimal average case errors $r_{n}$ of $n$ nonadaptive sample points can be bounded by two convex sequences $\alpha_{n}$ and $\beta_{n}$ such that $\alpha_{n} \leq r_{n} \leq \beta_{n}$ and $\alpha_{n}=\Theta\left(\beta_{n}\right)$. In our case, $r_{n}=\Theta\left(n^{-2}(\log n)^{d-1}\right)$ and we can take $\alpha_{n}=c_{1} n^{-2}(\log n)^{d-1}, \beta_{n}=c_{2} n^{-2}(\log n)^{d-1}$ for some positive 
constants $c_{1}$ and $c_{2}$. Thus, adaption does not help much and

$$
\operatorname{comp}^{\text {avg }}\left(\varepsilon, C_{d}\right)=\Omega\left(\mathbf{c} \varepsilon^{-1}\left(\log \varepsilon^{-1}\right)^{(d-1) / 2}\right) \text {. }
$$

This completes the proof of (i) and the rest follows easily from (i).

\section{Final Remarks}

A. The definition (4) of optimal sample points is not fully constructive due to the unspecified constant $t^{*}$ in (3). It would be interesting to determine the constant $t^{*}$ explicitly. On the other hand, if one takes the classical Hammersley points

$$
z_{k}=\left[k n^{-1}, \phi_{p_{1}}(k), \ldots, \phi_{p_{d-1}}(k)\right], \quad k=1, \ldots, n,
$$

then Halton proved, see e.g. [5], that even the $L_{\infty}$ discrepancy of the points $z_{k}$ is of order $n^{-1}(\log n)^{d-1}$. Then the approximation $U(f)=n^{-1} \sum_{k=1}^{n} f\left(x_{k}\right)$ with $x_{k}=\overrightarrow{1}-z_{k}$ has average case error at most $\varepsilon$ provided that $n=\Theta\left(\varepsilon^{-1}\left(\log \varepsilon^{-1}\right)^{d-1}\right)$.

Observe that the definition of $x_{k}$ (as well as $x_{k}^{*}$ in (4)) depends on the total number $n$ of samples. Sometimes it is better to use an infinite sequence $\left\{\tilde{x}_{k}\right\}$ in which the definition of $\tilde{x}_{k}$ does not depend on the specific value of $n$. For instance $\tilde{x}_{k}$ can be given by

$$
\tilde{x}_{k}=\overrightarrow{1}-\left[\phi_{p_{1}}(k), \phi_{p_{2}}(k), \ldots, \phi_{p_{d}}(k)\right], \quad k=1,2, \ldots,
$$

where $p_{1}, \ldots, p_{d}$ are the first $d$ prime numbers. The $L_{2}$ discrepancy of $n$ points $\overrightarrow{1}-\tilde{x}_{k}$ is at most of order $n^{-1}(\log n)^{d}$, see [5], and therefore the approximation $U(f)=n^{-1} \sum_{k=1}^{n} f\left(\tilde{x}_{k}\right)$ has the average case error at most $\varepsilon$ provided that $n=\Theta\left(\varepsilon^{-1}\left(\log \varepsilon^{-1}\right)^{d}\right)$.

In both cases, we see that the use of $x_{k}$ or $\tilde{x}_{k}$ leads to a slight increase in the average case cost. More precisely, instead of the minimal average case cost $\Theta\left(\mathbf{c} \varepsilon^{-1}\left(\log \varepsilon^{-1}\right)^{(d-1) / 2}\right)^{\prime}$, we approximate multivariate integrals at average case cost $O\left(\mathbf{c} \varepsilon^{-1}\left(\log \varepsilon^{-1}\right)^{d-1}\right)$ using the sample points $x_{k}$, or $O\left(c \varepsilon^{-1}\left(\log \varepsilon^{-1}\right)^{d}\right)$ using the sample points $\tilde{x}_{k}$.

B. We have shown that the minimal number of sample points $n(\varepsilon)$ to guarantee average case error $\varepsilon$ is of order $\varepsilon^{-1}\left(\log \varepsilon^{-1}\right)^{(d-1) / 2}$ with the constant in the $\Theta$ notation dependent only on $d$. An open problem is to ascertain if there exists a constant $\alpha_{d}$ and, if so, to find $\alpha_{d}$ such that

$$
n(\varepsilon)=\alpha_{d} \varepsilon^{-1}\left(\log \varepsilon^{-1}\right)^{(d-1) / 2}(1+o(1) \quad \text { as } \varepsilon \rightarrow 0 .
$$


C. It would be interesting to extend the results of this paper for smoother classes of functions equipped with folded Wiener sheet measures.

D. Do similar relations to the one we have utilized here between discrepancy and multivariate integration, hold for other problems such as approximation of functions of $d$ variables?

\section{ACKNOWLEDGMENTS}

I am grateful to J. F. Traub for valuable comments and help during the preparation of this paper. I also appreciate remarks from M. Kon, G. W. Wasilkowski, and A. G. Werschulz.

\section{REFERENCES}

1. G. S. Kimeldorf and G. Wahba, A correspondence between Bayesian estimation on stochastic processes and smoothing by splines, Ann. Math. Stat. 41 (1970), 495-502.

, Spline functions and stochastic processes, Sankhya Ser. A 32 (1970), 173-180.

3. D. Lee and G. W. Wasilkowski, Approximation of linear functionals on a Banach space with a Gaussian measure, J. Complexity 2 (1986), 12-43.

4. C. A. Micchelli and G. Wahba, Design problems for optimal surface interpolation, Approximation Theory and Applications (Z. Ziegler, ed.), Academic Press, New York, 1981, pp. 329-347.

5. H. Niederreiter, Quasi-Monte Carlo methods and pseudo-random numbers, Bull. Amer. Math. Soc. (N.S.) 84 (1978), 957-1041.

, Quasi-Monte Carlo methods for multidimensional numerical integration, Numerical Integration III, International Series of Numerical Mathematics (H. Braß and G. Hämmerlin . ed.), vol. 85, Birkhäuser-Verlag, Basel, 1988, pp. 157-171.

7. E. Novak, Deterministic and stochastic error bounds in numerical analysis, vol. 1349, Lectures Notes in Math. Springer-Verlag, Berlin, 1988.

8. A. Papageorgiou, On average case complexity, Ph. D. thesis, Dept. of Computer Science, Columbia University, 1989.

9. A. Papageorgiou and G. W. Wasilkowski, On the average complexity of multivariate problems, J. Complexity 6 (1990), 1-23.

10. K. F. Roth, On irregularities of distribution, Mathematika 1 (1954), 73-79. , On irregularities of distribution, IV, Acta Arith. 37 (1980), 67-75.

12. J. Sacks J. and D. Ylvisaker, Designs for regression with correlated errors, Ann. Math. Stat. 37 (1966), 68-89.

Designs for regression problems with correlated errors; many parameters, Ann. Math. Stat. 39 (1968), 49-69.

, Designs for regression problems with correlated errors III, Ann. Math. Stat. 41 (1970), 2057-2074.

15. J. Sacks and D. Ylvisaker, Statistical design and integral approximation, Proc. 12th Bienn. Semin. Can. Math. Cong. (1970), 115-136. 
16. A. V. Suldin, Wiener measure and its applications to approximation methods, I and II, (in Russian), Izv. Vyssh. Ucheb. Zaved. Mat. 13, 18 (1959, 1960), 145-158, 165-179.

17. J. F. Traub, G. W. Wasilkowski, and H. Woźniakowski, Information-based Complexity, Academic Press, New York, 1988.

18. G. Wahba, On the regression design problem of Sacks and Ylvisaker, Ann. Math, Stat. 42 (1971), 1035-1043.

19. G. W. Wasilkowski, Information of varying cardinality, J. Complexity 2 (1986), 204-228.

20. D. Ylvisaker, Designs on random fields, A Survey of Statistical Design and Linear Models (J. Srivastava, ed.), North-Holland, 1975, pp. 593-607.

Department of Computer Science, Columbia University and Institute OF INFORMATICS, UNIVERSITY OF WARSAW

E-mail address: henryk@cs.columbia.edu 